\title{
Anticoagulant Therapy Overview
}

\section{GEORGE A. FRITSMA}

\section{LEARNING OBJECTIVES}

1. Recount the history of heparin, Coumadin, and hirudin

2. Summarize the function of the anti-Xa drugs, vitamin $\mathrm{K}$ antagonists, and direct thrombin inhibitors.

3. List two oral anticoagulants that have been cleared by the US FDA since 2009 .

ABBREVIATIONS: APTT or PTT-activated partial thromboplastin time; CAD - coronary artery disease; DTI - direct thrombin inhibitor; DVT - deep venous thrombosis; FDA - US Food and Drug Administration; HIT-heparin-induced thrombocytopenia with thrombosis; INR - international normalized ratio; LMWH low molecular weight heparin; PE - pulmonary embolism; PT - prothrombin time; RI - reference interval; RUO - research use only; TPA - tissue plasminogen activator; UFH - unfractionated heparin; VKORC - vitamin K epoxide reductase; VKA - vitamin $\mathrm{K}$ antagonist; VTE - venous thromboembolism.

INDEX TERMS: Anticoagulants, heparin, fondaparinux, rivaroxaban, apixaban, Coumadin, warfarin, lepirudin, argatroban, bivalirudin, dabigatran, prothrombin time, activated partial thromboplastin time, anti-Xa heparin assay, atrial fibrillation, thrombosis, thromboembolic disease, coronary artery disease.

\section{Clin Lab Sci 2013;26(1):39}

George A. Fritsma MS MT (ASCP), The Fritsma Factor, Your Interactive Hemostasis Resource ${ }^{S M}$, Fritsma \& Fritsma LLC, Birmingham, AL

Address for Correspondence: George A. Fritsma MS MT (ASCP), The Fritsma Factor, Your Interactive Hemostasis Resource $^{S M}$, Fritsma \& Fritsma LLC, 153 Redwood Drive, Birmingham, AL 35173, 205-655-0687, George@ fritsmafactor.com.

Anticoagulants do not thin the blood, even though we call them "blood thinners." Blood viscosity is a function of hematocrit and, in leukemia, white blood cell burden, but not plasma protein concentration or enzyme activity. Although thrombolytic "clotbusters" like tissue plasminogen activator (TPA) or streptokinase can dissolve clots, anticoagulants cannot, another popular misconception. Anticoagulants can only prevent thrombus formation or extension by reducing plasma thrombin activity, the basis for their effectiveness. Though this may seem like a limitation, anticoagulants save many lives and promote recovery following a thrombotic (clotting) event. ${ }^{2}$

Anticoagulant treatment is either prophylactic or therapeutic. Prophylactic anticoagulation includes the prevention of ischemic stroke in people with chronic atrial fibrillation or artificial heart valves, or the prevention of venous thromboembolic (VTE) disease in the form of deep venous thrombosis (DVT, clots in major leg veins) or pulmonary emboli (PE, clots in lung vasculature), subsequent to orthopedic surgery, neurosurgery, or during a complicated pregnancy. ${ }^{3}$ Therapeutic anticoagulation, which usually implies higher doses than prophylactic anticoagulation, is used in patients with current VTE disease or those with coronary artery disease (CAD) complicated by cardiac insufficiency. ${ }^{4}$

Anticoagulants are a subset of a family of drugs called antithrombotics, which include the oral antiplatelet drugs such as aspirin and clopidogrel (Plavix); the intravenous antiplatelet membrane glycoprotein receptor drugs, abciximab, eptifibatide, and tirofiban; and the thrombolytics.

This series does not address the antiplatelet drugs or the thrombolytics. In this series we first address the anti-Xa anticoagulants, heparin and its analogues; next, the historical mainstay Coumadin, our only vitamin $\mathrm{K}$ antagonist (VKA); and last, the direct thrombin inhibitors (DTIs), represented by their original synthetic hirudin (lepirudin), analogue of a leech saliva component, now discontinued; bivalirudin, an 
abbreviated form of lepirudin; argatroban; and the first oral DTI, dabigatran, released in 2010 for stroke prevention in chronic atrial fibrillation.

\section{Heparin and The Parenteral Anti-Xa Anticoagulants} In 1916, Jay McLean and William Henry Howell extracted heparin, the first anticoagulant, from dog liver. ${ }^{5}$ They recognized its anticoagulant properties and derived its name from its liver origin. In 1935, Erik Jorpe of the Karolinska Institut, Stockholm, accurately described heparin's chemical structure, whereupon the University of Toronto Connaught Medical Research Laboratories produced a heparin salt safe for human therapy. Heparin was cleared as a therapeutic by the newly formed United States Food and Drug Administration (FDA) in 1937.

Heparin, now called standard or unfractionated heparin (UFH), is a linear glycosaminoglycan composed of an average of sixty sugar molecules, one of the most negatively charged molecules in nature. UFH mimics endothelial cell heparan sulfate, is extracted from porcine stomach and intestinal linings, and since 1937 it has been administered intravenously to successfully prevent and treat both arterial and venous thrombosis. The pharmacokinetics of UFH are idiosyncratic, thus heparin therapy requires frequent laboratory monitoring until it is discontinued. ${ }^{6}$ This is because UFH differentially binds patients' endothelial cell surface molecules and a variety of patients' plasma proteins.

In 1993 the US FDA cleared low molecular weight heparin (LMWH), another glycosaminoglycan, which is derived from UFH through enzymatic or chemical depolymerization. LMWH's predictable dose-response properties make it safer and easier to administer than UFH. LMWH, injected subcutaneously, is available for either prophylaxis or treatment of venous or arterial thrombosis, and requires no recurrent laboratory monitoring. The most commonly prescribed brand of LMWH in the US is Lovenox ${ }^{\circledR}$, generic name enoxaparin, marketed by Aventis, Inc.

Pharmaceutical manufacturers prefer to develop anticoagulants that require no laboratory monitoring, a significant convenience for the patient and an obvious marketing advantage. Consequently, drug monitoring is not included in clinical trials. LMWH was the first of the anticoagulants possessing stable pharmacokinetics, and when it was released, laboratories had no FDAcleared means to measure its plasma concentration. Although we need not regularly monitor LMWH as we do its more dangerous parent, UFH, we often must test for its plasma concentration in anyone who possesses a coagulopathy or osmotic imbalance. These include pregnancy, patients with renal or hepatic disease, cancer, diabetes, or a previous thrombotic event; the morbidly obese, the exceptionally slender, or children. We also required a means to check for compliance or to identify the cause of hemorrhage for emergency department patients unable to name their drug. The in vitro diagnostics (IVD) industry scrambled to develop an effective laboratory test, the anti-Xa heparin assay, which is now in common use, because the traditional assay that was used to monitor UFH, the activated partial thromboplastin time (APTT, PTT) was insensitive. Laboratory directors and IVD manufacturers are repeatedly challenged to find clinically effective means for testing new anticoagulants as they are released, and are currently scrambling for ways to measure the new anticoagulants dabigatran and rivaroxaban.

In 2001, the US FDA cleared danaparoid sodium, a judiciously titrated mixture of heparan sulfate, dermatan sulfate, and chondroitin sulfate. Danaparoid sodium is the first synthetic form of heparin. It has never been successfully marketed in the United States, but remains available in Northern Europe as Orgaran ${ }^{\circledR}$, marketed by Schering-Plough, Inc.

Fondaparinux, a synthetic that mimics a specific heparin pentasaccharide sequence and is employed for the same indications as LMWH, was released in 2001. Fondaparinux is marketed as $\operatorname{Arixtra}^{\circledR}$ by GlaxoSmithKline and has better efficacy and safety than LMWH. A generic form of fondaparinux was cleared in 2011 after Glaxo's patent expired. UFH, LMWH, danaparoid, and fondaparinux all raise the effect of plasma antithrombin upon the serine proteases of the coagulation cascade, especially thrombin (IIa) and activated coagulation factor $\mathrm{X}$, or Xa.

Rivaroxaban, the first of the oral direct anti-Xa anticoagulants, was approved for the prevention of stroke in patients with atrial fibrillation in November, 2011, and apixaban, the second oral direct anti-Xa anticoagulant was cleared for the same purpose in 
December, 2012. Rivaroxaban and apixaban act directly to inhibit $\mathrm{Xa}$ activity, bypassing antithrombin. Rivaroxaban is marketed as Xarelto ${ }^{\circledR}$ by Janssen Pharmaceuticals, Inc, and apixaban is marketed as Eliquis ${ }^{\circledR}$ by Bristol-Myers-Squibb, once cleared. Both can be monitored using a modification of the anti-Xa heparin assay, however the modification requires new FDA clearance and is currently under review.

Read about the means for laboratory monitoring of $\mathrm{UFH}$, LMWH, rivaroxaban, and apixaban in the accompanying article, Monitoring the Anti-Xa Anticoagulants, from Heparin to Eliquis.

\section{Vitamin K Antagonist: Coumadin}

The familiar history of the vitamin $\mathrm{K}$ antagonist (VKA) Coumadin begins in 1921 when Canadian veterinary pathologist Frank Schofield proved that an epidemic of fatal cattle hemorrhages in the Northwest US and Southwest Canada was caused by fungus-infected (spoiled) sweet clover, a component of silage. In 1933, Dr. Karl Link, University of Wisconsin, assigned graduate students the task of deriving and synthesizing the component responsible for the loss of cattle, and in 1940, Wisconsin graduate Harold Campbell first characterized dicoumarol, a synthetic analogue of coumarin, the compound that produces the pleasant odor of newly mowed hay or grass. Spoiled sweet clover produces 4-hydroxycoumarin, the anticoagulant responsible for the bleeding disorder in cattle, mimicked by the synthetic dicoumarol.

Link and his chemists continued to develop dicoumarol, and by 1948 were awarded a patent for warfarin, which soon became popular as a commercial rodent poison. Warfarin is named for the Wisconsin Alumni Research Foundation (WARF), which funded Link's research and held the patent.

In 1951 a soldier attempted suicide by ingesting rat poison, and his symptoms were reversed using vitamin K. By 1954 the medical version of warfarin, Coumadin, was released by the US FDA, and in 1955 the drug gained fame as President Dwight D. Eisenhower was prescribed Coumadin following a heart attack. Coumadin was intended to prevent recurrence of myocardial infarction and was used in this capacity until approximately 2000 when it was largely replaced by more effective antiplatelet therapy.
The term "vitamin K antagonist" is a biochemical misnomer. Instead, Coumadin inhibits the liver enzyme vitamin $\mathrm{K}$ epoxide reductase (VKORC) that normally reduces oxidized vitamin $\mathrm{K}$. Reduced vitamin $\mathrm{K}$ catalyzes the $\gamma$-carboxylation of coagulation factors II (prothrombin), VII, IX, and X and coagulation control proteins $\mathrm{C}, \mathrm{S}$, and $\mathrm{Z}$. Vitamin $\mathrm{K}$ becomes oxidized in the process of $\gamma$-carboxylation, and requires VKORC to become reduced and reenter the reaction. Inhibited by Coumadin, the liver produces ineffective "des-carboxy" forms of II, VII, IX, X, C, S, and Z, thereby reducing thrombin activity and thrombus formation.

Coumadin is the most popular, yet the most dangerous of the anticoagulants. It must be monitored at least every four weeks for the duration of therapy, often lifelong. The clinical laboratory offers the prothrombin time (PT), whose results in seconds are exponentially modified by the international sensitivity index, a measure of thromboplastin reagent sensitivity, to generate the international normalized ratio (INR). ${ }^{7}$ An overdose produces hemorrhage, inadequate dosing leads to a repeat adverse thrombotic event, and the therapeutic range, INR 2.0-3.0, is narrow. The indications, mechanism of action, dosing, monitoring, and limitations of Coumadin are discussed in the accompanying article, Coumadin, the Original Oral Anticoagulant.

\section{Direct Thrombin Inhibitors}

The "youngest" class of anticoagulants, direct thrombin inhibitors (DTIs) bind and inhibit thrombin without involving any other coagulation protein. ${ }^{8}$ Hirudin, a component of Hirudo medicinalis (medicinal leech) saliva, was first described as an anticoagulant by Haycraft way back in 1884, was chemically characterized in 1976, and synthesized as lepirudin by Berlex, Inc in the 1990s. Lepirudin (Refludan ${ }^{\circledR}$ ), a 65 amino acid polypeptide, was cleared by the FDA in 1998. It was indicated as a substitute for UFH in patients who had developed heparin-induced thrombocytopenia with thrombosis (HITT, HIT). Largely replaced by the oligopeptide bivalirudin (Angiomax ${ }^{\circledR}$ ), released in 2006, lepirudin's European producer terminated its production on May 31, 2012.

A more popular DTI, GlaxoSmithKline's small molecule Argatroban ${ }^{\circledR}$, was released in 2002 to function, like lepirudin, as a heparin substitute in HIT. 
Argatroban and bivalirudin are administered intravenously and are used in conjunction with aspirin and clopidogrel.

In 2004 the FDA rejected ximelagatran (AstraZenaca's Exanta $\left.^{\circledR}\right)$, an oral DTI with considerable promise. ${ }^{10}$ Exanta would have been the eagerly awaited first new oral anticoagulant since Coumadin, however it failed to clear because it caused elevated liver enzymes in 5-10\% of clinical trial subjects. Although it had cleared in Northern Europe, the manufacturer withdrew the drug in 2006 as it appeared to produce chronic liver disease in a few patients.

In 2010, the US FDA cleared Boehringer Ingelheim's oral DTI, dabigatran etexilate $\left(\right.$ Pradaxa $^{\circledR}$ ), the first successful oral anticoagulant since Coumadin. Pradaxa is currently indicated for prevention of ischemic stroke in atrial fibrillation, although it may later be cleared for VTE prophylaxis in orthopedic surgery and neurosurgery. As was the case with enoxaparin and fondaparinux, no laboratory tests are currently cleared to detect and measure DTI plasma concentrations, hence IVD developers are feverishly conducting clinical trials for a variety of potential assays, as described in our accompanying article, Monitoring the Direct Thrombin Inhibitors.

Medical laboratory science is ever changing, and nowhere as rapidly as in the field of anticoagulant therapy. After 50 years using the same two anticoagulants, clinicians now have a surfeit of choices, with several more in the works. Perhaps by the time these articles are published, apixaban is recently cleared for prophylaxis, and perhaps by the time these articles are published, some new DTI or anti-Xa anticoagulant will have reached the FDA for review. Further, assays currently categorized as research use only (RUO) will be available in automated or kit form, making it possible for acute care facilities to conveniently assay the new drugs.

\section{REFERENCES:}

1. Fritsma GA. Monitoring antithrombotic therapies. In Rodak BF, Fritsma GA, Keohane EM. Hematology; Clinical Principles and Applications, 4th Edition. Elsevier, St. Louis, 2012.

2. Francis CW. Antithrombotic agents. In Kitchens CS, Alving BM, Kessler CM. Consultative Hemostasis and Thrombosis, 2nd Edition. Elsevier, St. Louis, 2007.

3. Tran HAM, Ginsberg JS. Chapter 116: Anticoagulant therapy for major arterial and venous thromboembolism. In Colman RW, Marder VJ, Clowes AW, George JN, Goldhaber SZ. Hemostasis and Thrombosis; Basic Principles and Clinical Practice, 5th Edition. LWW Philadelphia 2006.

4. Marques MB, Fritsma GA. Quick Guide to Coagulation Testing 2nd Edition. AACC Press, Washington DC, 2009.

5. Marcum JA. The origin of the dispute over the discovery of heparin. J Hist Med Allied Sci 2000;55:37-66.

6. Garcia DA, Baglin TP, Weitz JI, Samama MM. Parenteral anticoagulants: antithrombotic therapy and prevention of thrombosis, 9th Edition. American College of Chest Physicians evidence-based clinical practice guidelines. Chest 2012;141;e24S-e43S.

7. Ageno W, Gallus AS, Wittkowsky A, Crowther M, Hylek EM, Palareti G. Oral anticoagulant therapy: antithrombotic therapy and prevention of thrombosis, 9th Edition. American College of Chest Physicians evidence-based clinical practice guidelines. Chest 2012;141;e44S-e88S.

8. Di Nisio M, Middeldorp S, Büller HR. Direct thrombin inhibitors. N Engl J Med 2005;353:1028-40.

9. Linkins L, Dans AL, Moores LK, Bona R, Davidson BL, Schulman S, Crowther M. Treatment and prevention of heparin-induced thrombocytopenai: antithrombotic therapy and prevention of thrombosis, 9th Edition. American College of Chest Physicians evidence-based clinical practice guidelines. Chest 2012;141;e495S-e530S.

10. Hirsh J, O'Donnell M, Eikelboom JW. Beyond unfractionated heparin and warfarin: current and future advances. Circulation 2007;116:552-60. 